ESAIM: PROCEEDINGS, November 2002, Vol.12, 48-54

M.Thiriet, Editor

\title{
COMPUTATIONAL MODELLING OF 1D BLOOD FLOW AND ITS APPLICATIONS.
}

\author{
V. Franke ${ }^{1}$, J. Peiró ${ }^{1}$, S. Sherwin ${ }^{1}$, K.PArker ${ }^{2}$, Wee Ling $^{3}$ And N. M. Fisk ${ }^{3}$
}

\begin{abstract}
Pressure and blood flow waveforms in the human body can be recorded using techniques such as sphygmomanometry and Doppler ultrasound. The presence of abnormal waveforms in arteries may indicate a pathological state. In this paper arterial wave patterns are modelled using a simplified one dimensional model for blood flow. The hyperbolic system of governing equations is discretised using the discontinuous Galerkin method. Results are presented for patterns of blood flow and pressure waves throughout a simplified arterial system consisting of the main 55 arteries. The method is also employed to determine unusual wave patterns in monochorionic twin pregnancies where an anastomosis between the twins is present across the placenta equator.
\end{abstract}

\section{Method}

As a simple approximation, the human arteries can be modelled as distensible tubes. Assuming static equilibrium in the radial direction of the cylindrical tube and averaging the velocity across the section, the system can be reduced to a one-dimensional problem. The equations representing continuity of mass and momentum can be written as

$$
\begin{aligned}
& \frac{\partial A}{\partial t}+\frac{\partial A u}{\partial x}=0 \\
& \frac{\partial u}{\partial t}+u \frac{\partial u}{\partial x}=-\frac{1}{\rho} \frac{\partial p}{\partial x}
\end{aligned}
$$

where the $x$ is the axial direction, $A=A(x, t)$ is the cross sectional area, $u=u(x, t)$ is the velocity of the fluid, averaged across the section and $\rho=$ const is the density of the blood. Also an expression between the pressure, p, and area, A, of the vessel can be specified as:

$$
p=p_{e x t}+\beta\left(\sqrt{A}-\sqrt{A_{0}}\right)
$$

\footnotetext{
1 Biomedical Flow Group, Department of Aeronautics,

Imperial College of Science, Technology and Medicine,

Prince Consort Road, London SW7 2BY, U.K.

e-mail: v.shenton@ic.ac.uk, j.peiro@ic.ac.uk, s.sherwin@ic.ac.uk, web page: http://www.ae.ic.ac.uk/staff/sherwin

2 Department of Bioengineering,

Imperial College of Science, Technology and Medicine.

3 Imperial College School of Medicine at Queen Charlotte's,

Hammersmith Hospital Site.
} 
where

$$
\beta=\frac{\sqrt{\pi} h_{0} E}{\left(1-\nu^{2}\right) A_{0}} .
$$

In this relationship $h_{0}$ and $A_{0}=A_{0}(x)$ denote the vessel wall thickness and cross-sectional area at the equilibrium state, $E=E(x)$ is the Young modulus, $\nu$ is the Poisson ratio (typically considered as $\nu=1 / 2$ the incompressible limit) and $p_{\text {ext }}$ is the external pressure.

The one-dimensional hyperbolic system, equations (1) and (2) can be expressed as an (A,u) system in a conservative form:

where

$$
\frac{\partial \boldsymbol{U}}{\partial t}+\frac{\partial \boldsymbol{F}}{\partial x}=0
$$

$$
\boldsymbol{U}=\left[\begin{array}{c}
U_{1} \\
U_{2}
\end{array}\right]=\left[\begin{array}{l}
A \\
u
\end{array}\right] \quad \boldsymbol{F}=\left[\begin{array}{c}
F_{1} \\
F_{2}
\end{array}\right]=\left[\begin{array}{c}
u A \\
\frac{u^{2}}{2}+\frac{p}{\rho}
\end{array}\right] .
$$

\section{Characteristic System}

Applying the chain rule to the pressure-area relationship, equation (3), and assuming that $\beta=\beta(x)$ and $A_{0}=A_{0}(x)$ we get:

where we note that

$$
\frac{\partial p}{\partial x}=\frac{\partial p}{\partial A} \frac{\partial A}{\partial x}+\frac{\partial p}{\partial \beta} \frac{\partial \beta}{\partial x}+\frac{\partial p}{\partial A_{0}} \frac{\partial A_{0}}{\partial x}
$$

$$
\frac{d p}{d A}=\frac{\beta}{2 \sqrt{A}}
$$

By simple manipulations the system of equations (1) and (2) can be written in a quasi-linear conservative form:

where

$$
\frac{\partial \boldsymbol{U}}{\partial t}+\boldsymbol{H} \frac{\partial \boldsymbol{U}}{\partial x}=\left[\begin{array}{l}
A \\
u
\end{array}\right]_{t}+\left[\begin{array}{cc}
u & A \\
c^{2} / A & u
\end{array}\right]\left[\begin{array}{l}
A \\
u
\end{array}\right]_{x}=\left[\begin{array}{l}
0 \\
f
\end{array}\right]
$$

$$
c^{2}=\frac{A}{\rho} \frac{\partial p}{\partial A}=\frac{\beta A^{1 / 2}}{2 \rho} \text { and } f=\frac{1}{\rho}\left[-\frac{\partial p}{\partial \beta} \frac{\partial \beta}{\partial x}-\frac{\partial p}{\partial A_{0}} \frac{\partial A_{0}}{\partial x}\right] .
$$

Matrix $\boldsymbol{H}$ has two real eigenvalues $\lambda_{1,2}=u \pm c$ and the corresponding eigenmatrix $\boldsymbol{Q}$ is

$$
\boldsymbol{Q}=\left[\boldsymbol{q}_{\mathbf{1}}, \boldsymbol{q}_{\mathbf{2}}\right]=\gamma\left[\begin{array}{cc}
A & -A \\
c & c
\end{array}\right]
$$

where $\gamma$ is an arbitary scaling factor.

If $\boldsymbol{H} \boldsymbol{Q}=\boldsymbol{Q} \boldsymbol{\Lambda}$ then the matrix $\boldsymbol{H}=\boldsymbol{Q} \boldsymbol{\Lambda} \boldsymbol{Q}^{-1}$, where $\boldsymbol{\Lambda}$ is the diagonal matrix formed by the eigenvalues of $\boldsymbol{H}$.

Substituting this into equation (5), assuming $f=0$, and premultiplying by $\boldsymbol{Q}^{-1}$ gives:

If there exists a quantity $\boldsymbol{W}=\boldsymbol{W}(\boldsymbol{U})$ such that

$$
\boldsymbol{Q}^{-1} \frac{\partial \boldsymbol{U}}{\partial t}+\boldsymbol{\Lambda} \boldsymbol{Q}^{-1} \frac{\partial \boldsymbol{U}}{\partial x}=0
$$

$$
\frac{\partial \boldsymbol{W}}{\partial \boldsymbol{U}}=\boldsymbol{Q}^{-1}
$$

then the characteristic variables can be determined by integrating the differential system:

$$
\boldsymbol{Q}^{-1}=\gamma\left[\begin{array}{cc}
\frac{1}{2 A} & \frac{1}{2 c} \\
-\frac{1}{2 A} & \frac{1}{2 c}
\end{array}\right]=\frac{\partial \boldsymbol{W}}{\partial \boldsymbol{U}}=\gamma\left[\begin{array}{ll}
\frac{\partial W_{1}}{\partial A} & \frac{\partial W_{1}}{\partial u} \\
\frac{\partial W_{2}}{\partial A} & \frac{\partial W_{2}}{\partial u}
\end{array}\right]
$$

A possible choice for $\gamma$ where the characteristic variables satisfy the Cauchy-Riemann condition would be $\gamma=2 c$, since A does not depend on $\mathrm{u}$. Therefore if the characteristic variables exist they must satisy: 


$$
\begin{aligned}
\frac{\partial W_{1}}{\partial A}=\frac{c}{A}, & \frac{\partial W_{1}}{\partial u}=1, \\
\frac{\partial W_{2}}{\partial A}=-\frac{c}{A}, & \frac{\partial W_{2}}{\partial u}=1
\end{aligned}
$$

Integrating the differential form in equations (10) and (11) we obtain:

$$
\begin{gathered}
W_{1}=u+\int_{A_{0}}^{A} \frac{c(A)}{A} d A=u+4 c=u+4 \sqrt{\frac{\beta}{2 \rho}} A^{1 / 4}, \\
W_{2}=u-\int_{A_{0}}^{A} \frac{c(A)}{A} d A=u-4 c=u-4 \sqrt{\frac{\beta}{2 \rho}} A^{1 / 4}
\end{gathered}
$$

Since $\beta>0$, we may write, as previously reported in [3], the variables $(A, u)$ in terms of $\left(W_{1}, W_{2}\right)$ as

$$
A=\left[\frac{\left(W_{1}-W_{2}\right)}{4}\right]^{4}\left(\frac{\rho}{2 \beta}\right)^{2} \quad u=\frac{\left(W_{1}+W_{2}\right)}{2} .
$$

Using the relation in equation (8), equation (7) can be transformed into a decoupled system of equations for the characteristic variables, which component wise reads

$$
\begin{aligned}
& \frac{\partial W_{1}}{\partial t}+\lambda_{1} \frac{\partial W_{1}}{\partial x}=0 \\
& \frac{\partial W_{2}}{\partial t}+\lambda_{2} \frac{\partial W_{2}}{\partial x}=0
\end{aligned}
$$

\section{Boundary Conditions}

The hyperbolic system requires us to impose one boundary condition at the inflow and the outflow, these are imposed through the characteristic system. At the inlet this can be either a velocity $u$ or area $A$. In vivo values can be obtained from methods such as Doppler ultrasound. At the outlet a reflection coefficient is applied to the waves exiting the vessels which is representative of a terminal resistance in the arterial network beyond the terminal vessels. More details are found in [5].

\section{Discontinuous Galerkin Method}

The wave propagation speeds in the large arteries are typically an order of magnitude higher than the average flow speeds, hence the characteristic system is subcritical (i.e. $\lambda_{1}>0$ and $\lambda_{2}<0$ ) for physiological conditions and will not produce shock waves in the system. If the solution remains smooth then high-order methods are particularly attractive due to the fast convergence of the phase and diffusion properties with order of the scheme [6]. The discontinuous Galerkin method is an attractive formulation for the high-order discretisation of these hyperbolic conservation laws as it propagates waves with minimum numerical diffusion and dispersion for many periods. Following the work of Cockburn and Shu [1] and Lomtev, Quillen and Karniadakis [4] we proceed as follows. 
To solve the system, equation (4), in a domain $\Omega=(a, b)$ discretised into a mesh of $N_{e l}$ elemental nonoverlapping regions $\Omega_{e}=\left(x_{e}^{l}, x_{e}^{u}\right)$, such that $x_{e}^{u}=x_{e+1}^{l}$ for $e=1, \ldots, N_{e l}$, and

$$
\bigcup_{e=1}^{N_{e l}} \bar{\Omega}_{e}=\bar{\Omega}
$$

we start by constructing the weak form of (4), i.e.

$$
\left(\frac{\partial \mathbf{U}}{\partial t}, \boldsymbol{\psi}\right)_{\Omega}+\left(\frac{\partial \mathbf{F}}{\partial x}, \boldsymbol{\psi}\right)_{\Omega}=0 \quad i=1,2
$$

where

$$
(\boldsymbol{u}, \boldsymbol{v})_{\Omega}=\int_{\Omega} \boldsymbol{u} \boldsymbol{v} d x
$$

is the standard $\boldsymbol{L}^{2}(\Omega)$ inner product. Decomposing the integral into elemental regions we obtain

$$
\sum_{e=1}^{N_{e l}}\left[\left(\frac{\partial \mathbf{U}}{\partial t}, \boldsymbol{\psi}\right)_{\Omega_{e}}+\left(\frac{\partial \mathbf{F}}{\partial x}, \boldsymbol{\psi}\right)_{\Omega_{e}}\right]=0
$$

Integrating the second term by parts leads to

$$
\sum_{e=1}^{N_{e l}}\left(\frac{\partial \mathbf{U}}{\partial t}, \boldsymbol{\psi}\right)_{\Omega_{e}}-\left(\mathbf{F}, \frac{d \boldsymbol{\psi}}{d x}\right)_{\Omega_{e}}+[\boldsymbol{\psi} \cdot \mathbf{F}]_{x_{e}^{l}}^{x_{e}^{u}}=0
$$

To get the discrete form of our problem we choose $\mathbf{U}$ to be in the finite space of $\boldsymbol{L}^{2}(\Omega)$ functions which are polynomial of degree $P$ on each element. We indicate an element of such space using the superscript $\delta$. We also note that $\mathbf{U}^{\delta}$ may be discontinuous across inter-element boundaries. However to attain a global solution in the domain $\Omega$ we need to allow information to propagate between the elemental regions. Information is propagated between elements by upwinding the boundary flux in the third term of equation (19). Denoting the upwinded flux as $\mathbf{F}^{u}$, the discrete weak formulation can now be written as

$$
\sum_{e=1}^{N_{e l}}\left(\frac{\partial \mathbf{U}^{\delta}}{\partial t}, \boldsymbol{\psi}^{\delta}\right)_{\Omega_{e}}-\left(\mathbf{F}\left(\mathbf{U}^{\delta}\right), \frac{d \boldsymbol{\psi}^{\delta}}{d x}\right)_{\Omega_{e}}+\left[\boldsymbol{\psi}^{\delta} \cdot \mathbf{F}^{u}\right]_{x_{e}^{l}}^{x_{e}^{u}}=0
$$

Following the traditional Galerkin approach, we choose the test function within each element to be in the same discrete space as the numerical solution $\mathbf{U}^{\delta}$. At this point if we define our polynomial basis and choose an appropriate quadrature rule we would now have a semi-discrete scheme. However, from an implementation point of view, the calculation of the second term can be inconvenient and consequently we choose to integrate this term by parts once more to obtain

$$
\sum_{e=1}^{N_{e l}}\left(\frac{\partial \mathbf{U}^{\delta}}{\partial t}, \boldsymbol{\psi}^{\delta}\right)_{\Omega_{e}}+\left(\frac{\partial \mathbf{F}\left(\mathbf{U}^{\delta}\right)}{\partial x}, \boldsymbol{\psi}^{\delta}\right)_{\Omega_{e}}+\left[\boldsymbol{\psi}^{\delta} \cdot\left[\mathbf{F}^{u}-\mathbf{F}\left(\mathbf{U}^{\delta}\right)\right]\right]_{x_{e}^{l}}^{x_{e}^{u}}=0
$$

We note that the information between elements is transmitted by the third boundary term as the difference between the upwinded and the local fluxes, $\left[\boldsymbol{\psi}^{\delta} \cdot\left[\mathbf{F}^{u}-\mathbf{F}\left(\mathbf{U}^{\delta}\right)\right]\right]_{x_{e}^{l}}^{x_{e}^{u}}$. This method can be considered as a penalty method with an automatic procedure for determining the penalty parameter. 
Finally we select our expansion bases to be a polynomial space of order $P$ and expand our solution on each element $e$ in terms of Legendre polynomials $L_{p}(\xi)$, i.e.

$$
\left.\mathbf{U}^{\delta}\right|_{\Omega_{e}}\left(x_{e}(\xi), t\right)=\sum_{p=0}^{P} L_{p}(\xi) \hat{\mathbf{U}}_{e}^{p}(t)
$$

where, following standard finite element techniques, we consider $\xi$ in the reference element $\Omega_{s t}=\{-1 \leq \xi \leq 1\}$ and introduce the elemental affine mapping

$$
x_{e}(\xi)=x_{e}^{l} \frac{(1-\xi)}{2}+x_{e}^{u} \frac{(1+\xi)}{2} .
$$

We note that the choice of discontinuous discrete solution and test functions allow us to decouple the problem on each element, the only link coming through the upwinded boundary fluxes. Legendre polynomials are particularly convenient because the basis is orthogonal with respect to the $\boldsymbol{L}^{2}\left(\Omega_{e}\right)$ inner product and equation (21) turns out to be equivalent to solving, componentwise, for all elements $e$

$$
J_{e} \frac{\partial \hat{U}_{i, e}^{p}}{\partial t}=-J_{e}\left(\frac{\partial F_{i}}{\partial x}, L_{p}\right)_{\Omega_{e}}-\left[L_{p}\left[F_{i}^{u}-F_{i}\left(\mathbf{U}^{\delta}\right)\right]\right]_{x_{e}^{l}}^{x_{e}^{u}}=0, \quad p=1, \ldots, P, \quad i=1,2
$$

where $J_{e}$ is the Jacobian of the elemental mapping, $J_{e}=\frac{1}{2}\left(x_{e}^{u}-x_{e}^{l}\right)$. To complete the discretisation we require a time integration scheme. Here we have adopted an Adams-Bashforth scheme. This upwinding process can also be used to impose the characteristic boundary conditions through the flux at the ends of the global domain $\Omega$.

\section{Results}

(a)

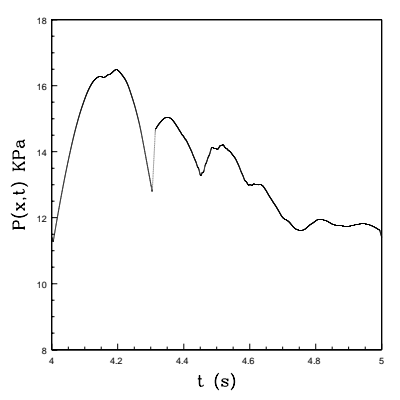

(c)

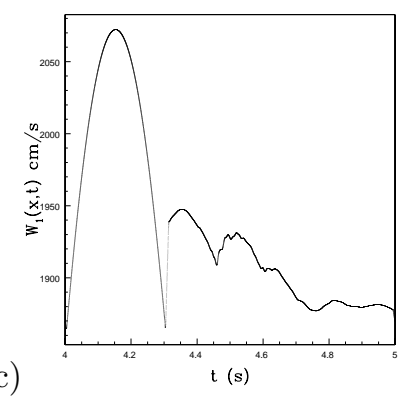

(b)

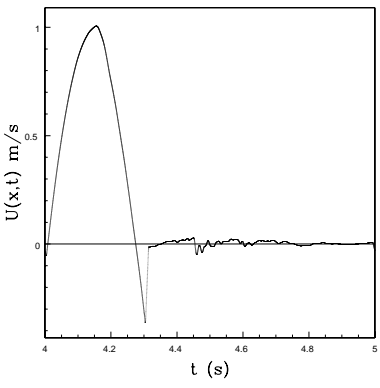

(d)

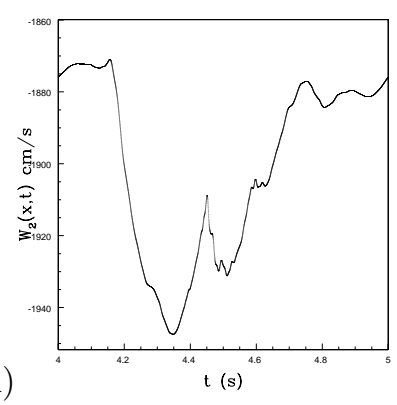

Figure 1.Time history plots of (a) pressure, (b) velocity and (c) and (d) the characteristic variables $W_{1}$ and $W_{2}$, respectively, taken at the mid-point of the ascending aorta. 
A simplified arterial network containing the 55 largest arteries in the human body was proposed and modelled using electric circuits in 1969 by Westerhof [10]. Modifications have been made to this data by Stergiopulos [7] and Wang and Parker [9]. We have used this modified data to compute the pulsatile one-dimensional blood flow through the arterial system. The flow in the 55 arteries is assumed initially to be at rest and a periodic half-sine wave is imposed through the $W_{1}$ waveform at the ascending aorta. The half-sine wave occupies the first 0.3 seconds of the cycle and for the rest of the cycle the heart acts as a reflector which has been modelled by setting $W_{1}=-W_{2}$. A lower terminal resistance has been used than in the Wang and Parker [9] model, because there was too much reflection from the terminal vessels and the waveforms did not appear to be physiologically correct.

The time history of pressure, velocity and the characteristic variables are shown in figure 5 over one cycle for the ascending aorta. The pressure waveform shows the characteristic diacrotic notch and the flow waveform shows a small period of flow reversal and then near zero flow for the rest of the cycle, which are similar to in-vivo measurements in the human body.

The one-dimensional model has also been applied to the calculation of waveforms in monochorionic twin pregnancies where twin fetuses share a single placenta. If there are anastomoses in the placenta between arteries and veins of the two fetoplacental circulations this can result in a transfer of blood from one fetus to the other with one fetus receiving too much blood to the detriment of the other twin. This medical condition is called twin-to-twin transfusion syndrome (TTTS) and can be fatal. Arterio-arterial anastomoses are present in $85 \%$ of monochorionic pregnancies, but this is not necessarily associated with a bad outcome. Indeed there is some evidence that the existence of these anastomoses may be protective, leading to a better outcome than in the TTTS cases with only arterio-venous anastomoses $[2,8]$.

(a)

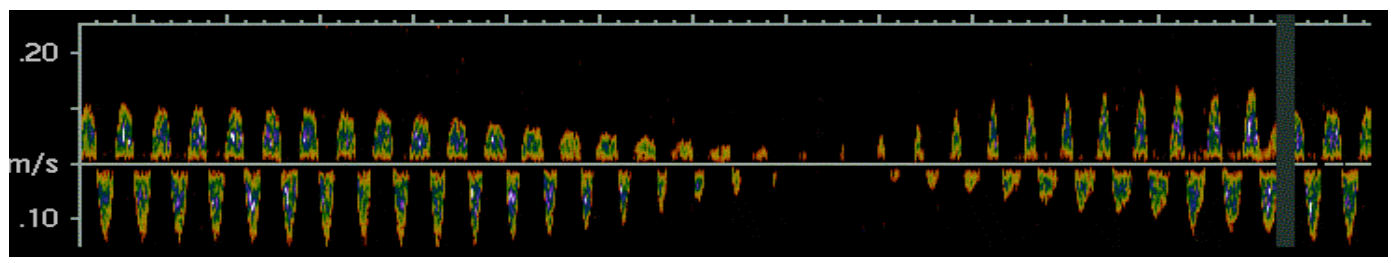

FiguRE 2.An ultrasound doppler flow waveform in a twin-to-twin anastomosis.

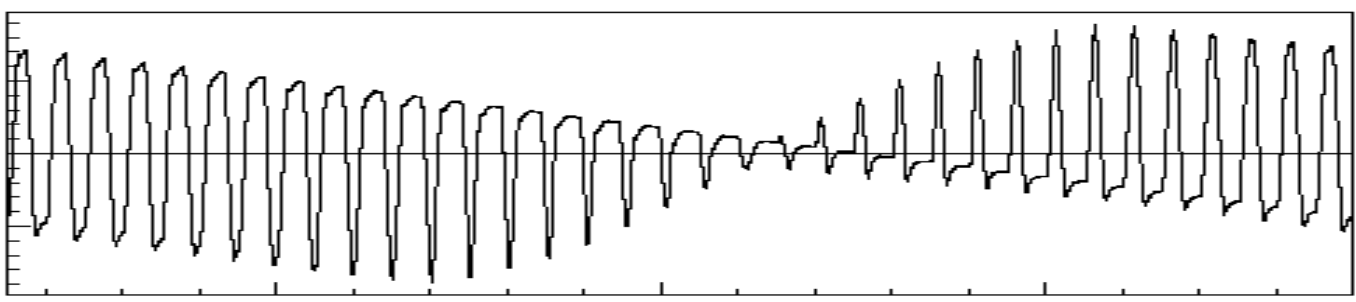

FiguRE 3.Predicted results from the one-dimensional mathematical model.

Figure 5 shows a Doppler ultrasound flow-probe waveform in an arterio-arterial anastomosis of a twin-to-twin pregnancy and figure 5 shows the results from our mathematical model. The fetuses often have slightly different heart rates, which produces a wave flow pattern that is repeated every few cycles. Also the flow alternates directions which is not seen in arteries in the normal placenta.

\section{Conclusion}

It has been shown that flow and pressure waveforms throughout different vascular networks can be predicted using a one-dimensional model. This model is inexpensive to compute and predicts accurately wave patterns and average velocities and pressures. Computer codes based on this approach could prove a valuable tool for assessing the suitability of medical interventions. 


\section{REFERENCES}

[1] B. Cockburn and C.W. Shu. TVB Runge-Kutta projection discontinous Galerkin finite element methods for conservation laws II general framework. Math. Comm., 52:411-435, 1989.

[2] M. L. Denbow, P. Cox, D. Talbert, and N. M. Fisk. Colour doppler energy insonation of placental vasculature in monochorionic twins: absent arterio-arterial anastomoses in association with twin-to-twin transfusion syndrome. Br. J. Obstet. Gynaecol., 105:760-765, 1998.

[3] L. Formaggia, F. Nobile, and A. Quarteroni. A one dimensional model for blood flow: application to vascular prosthesis. In I. Babuska, T. Miyoshi, and P.G. Ciarlet, editors, Mathematical Modeling and Numerical Simulation in Continuum MEchanics, volume 19 of Lecture Notes in Computational Science and Engineering, pages 137-153, Berlin, 2002. Springer-Verlag.

[4] I. Lomtev, C.W. Quillen, and G. Karniadakis. Spectral/ $h p$ methods for viscous compressible flows on unstructured $2 \mathrm{~d}$ meshes. J. Comp. Phys., 1998.

[5] S. J. Sherwin, L. Formaggia, J. Peiro, and V. Franke. Computational modelling of 1d blood flow with variable mechanical properties and its application to the simulation of wave propagation in the human arterial system. Submitted to Int. J. Numer. Meth. Fluids, 2002.

[6] S.J. Sherwin. Dispersion analysis of the continuous and discontinuous Galerkin formulations. In International Symposium on Discontinuous Galerkin Methods, 1999. Newport, RI.

[7] N. Stergiopulos and D.F. Young. Computer simulation of arterial flow with applications to arterial and aortic stenoses. $J$. Biomech., 25(12):1477-1488, 1992.

[8] M. J. O. Taylor, M. L. Denbow, S. Tanawattanacharoen, C. Gannon, P. Cox, and N. M. Fisk. Doppler detection of arterioarterial anastomoses in monochorionic twins: feasibility and clinical application. Hum. Reprod., 15:1632-1636, 2000.

[9] J.J. Wang and K.H. Parker. Wave propagation in a model of the arterial circulation. Submitted to J. Biomech., 2002.

[10] N. Westerhof, F. Bosman, C.J. De Vries, and A. Noordergraaf. Analog studies of the human systemic arterial tree. J. Biomech., 2:121-143, 1969. 